February 2006

PUPT-2192

YITP-SB-06-04

\title{
Double-Trace Deformations, Mixed Boundary Conditions and Functional Determinants in AdS/CFT
}

\author{
Thomas Hartman \\ Jefferson Physical Laboratory \\ Harvard University, Cambridge, MA 02138, USA \\ LEONARDO RASTELLI \\ C. N. Yang Institute for Theoretical Physics \\ Stony Brook University \\ Stony Brook, NY 11794, USA
}

\begin{abstract}
According to the AdS/CFT dictionary, perturbing the large $N$ boundary theory by a relevant double-trace deformation of the form $f \mathcal{O}^{2}$ corresponds in the bulk to imposing "mixed" boundary conditions for the field dual to $\mathcal{O}$. In this note we address various aspects of this correspondence. The change $c_{U V}-c_{I R}$ of the central charge between the UV ad IR fixed points is known from explicit calculations (hep-th/0210093, hep-th/0212138) to be exactly the same in the bulk and in the boundary theories. By comparing the appropriate bulk and boundary functional determinants, we give a simple "kinematic" explanation for this universal agreement. We also clarify the prescription for computing AdS/CFT correlators with $\Delta_{-}$boundary conditions.
\end{abstract}




\section{Introduction}

The purpose of this note is to give a simple explanation for a successful prediction of the AdS/CFT correspondence, concerning the renormalization group flow triggered in a large N CFT by a "double-trace" perturbation [1, 2, 3]. ${ }^{1} \mathrm{~A}$ $d$-dimensional conformal field theory perturbed by a relevant deformation of the form $f \mathcal{O}^{2}$, where $\mathcal{O}$ is a "single-trace" operator of dimension $\Delta_{-}<d / 2$, flows in the IR to another conformal fixed point [1. At large $N$, the IR theory is simply related to the UV theory - by a Legendre transformation with respect to the source for the operator $\mathcal{O}[3]$. In particular the conformal dimension of $\mathcal{O}$ flows from $\Delta_{-}$in the UV to $\Delta_{+}=d-\Delta_{-}$in the IR. This phenomenon has a holographic counterpart in $(d+1)$-dimensional Anti-de Sitter space, as the flow between different boundary conditions for the bulk field $\phi$ dual to $\mathcal{O}[1$. The boundary conditions preserve $\mathrm{AdS}$ isometries (i.e., conformal invariance) only at the extrema of the flow, where they correspond to the two roots $\Delta_{ \pm}$in the usual AdS/CFT formula $\Delta(\Delta-d)=m^{2}, m$ being the mass of the scalar field $\phi$. The variation of the central charge $c_{U V}-c_{I R}$ is a next-to-leading effect (of order $O(1)$ ) in the large $N$ expansion. It can be determined in the boundary CFT by an explicit mode sum [3], and in the bulk AdS theory by a one-loop evaluation of the effective potential for $\phi[2]$. The two calculations, while superficially quite different, are in perfect agreement. What is remarkable is the universality of this agreement, which holds for arbitrary spacetime dimension $d$, arbitrary conformal dimension $\Delta_{-}$, and independently of supersymmetry [3]. This seems to call for a more direct explanation.

Indeed, we find that the bulk and boundary calculations can be cast in a way that makes their equivalence manifest. Both in the boundary and in the bulk, evaluation of the central charge amounts to isolating the logarithmically divergent term of the partition function - this is a UV divergence in the boundary, and an IR divergence in the bulk, consistent with the familiar UV/IR connection. The bulk and boundary partition functions are appropriate functional determinants, evaluated respectively in the space of bulk and boundary fields. In essence, we are able to write the bulk functional determinant in a way that makes it obviously identical to the boundary functional determinant. Our methods should be applicable to more general backgrounds, for example AdS black hole backgrounds,

\footnotetext{
${ }^{1}$ Multitrace deformations were introduced in the context of AdS/CFT in [4. The correspondence between multitrace deformations of the boundary theory and boundary conditions in the bulk was understood in [1, 5] and further explored in e.g. 6, 7, 8, 9, 10, 2, 11, 3, 12, 13, 14, 15, 16, 17, 18, 19.
} 
where the bulk partition function corresponds to the thermal partition function of the boundary theory. They may also shed light on "designer gravity" [20], where a similarly universal bulk/boundary correspondence should find a more intrinsic explanation. Our result is similar in spirit to the recent understanding of " $\tau_{R R}$-minimization" [21] as the boundary dual of the bulk procedure of " $Z$ minimization" [22]: once the dust settles, the bulk and boundary calculations are seen to be isomorphic step by step. Of course, not all entries of the AdS/CFT dictionary are explained by such direct, purely kinematic mechanisms. But when they do, it seems worthwhile to spell out these mechanisms in detail.

We also take the opportunity to clarify the prescription to compute AdS/CFT correlators with the $\Delta_{-}$choice of boundary conditions. In section 5 we explain in diagrammatic terms how the $\Delta_{-}$boundary conditions for the AdS propagators lead automatically to the Legendre transform recipe postulated in [23. Finally, an appendix reviews the bulk calculation of Gubser and Mitra [2, with a slight modification of the regulator method, which allows us to check the assumption in [2] that $c_{U V}=c_{I R}$ at the Breitenlohner-Freedman [24] bound.

\section{Boundary: RG flow triggered by a double-trace deformation}

To make this paper self-contained, we briefly review in this section the field theoretic analysis of Gubser and Klebanov [3]. Consider the (Euclidean) partition function of a $d$-dimensional CFT, in the presence of the double-trace deformation $\frac{f}{2} \mathcal{O}^{2}$, and with a source $J$ for the single-trace operator $\mathcal{O}$,

$$
Z_{f}[J]=\left\langle\exp \left(-\int \frac{f}{2} \mathcal{O}^{2}+\int J \mathcal{O}\right)\right\rangle_{0}
$$

Here $\langle\ldots\rangle_{0}$ denotes a correlator in the unperturbed CFT. In order for the deformation to be relevant, the conformal dimension of $\mathcal{O}$ in the unperturbed theory is assumed to be $\Delta_{-} \equiv \frac{d}{2}-\nu$, with $\nu>0$. (Unitarity implies the lower bound $\Delta_{-} \geq \frac{d}{2}-1$, or $\nu \leq 1$.) The deformation parameter $f$ has engineering dimension $2 \nu>0$ and we expect that $f \rightarrow \infty$ in the IR. In fact, the theory can be analyzed exactly in the large $N$ limit, all along the $\mathrm{RG}$ flow, using a variant of the Hubbard-Stratonovich method. This consists of introducing an auxiliary field $\sigma$, modifying the action as

$$
S \rightarrow S-\frac{1}{2 f} \int(\sigma+f \mathcal{O})^{2} .
$$

The additional term does not change the physics - the non-dynamical $\sigma$ field can be integrated out giving back the original theory. The partition function (2.1) 
can be written as

$$
Z_{f}[J]=\sqrt{\operatorname{det}\left(-f^{-1} \mathbf{1}\right)} \int \mathcal{D} \sigma\left\langle\exp \int\left(\frac{\sigma^{2}}{2 f}+(J+\sigma) \mathcal{O}\right)\right\rangle_{0} .
$$

Dropping subleading terms in $1 / N$,

$$
\left\langle\exp \int(\sigma+J) \mathcal{O}\right\rangle_{0} \cong \exp \left(\frac{1}{2}\left\langle\left(\int(\sigma+J) \mathcal{O}\right)^{2}\right\rangle_{0}\right) .
$$

To write a closed form expression for $Z_{f}[J]$ it is convenient to introduce the operator $\hat{G}$, defined in position space as the convolution with the (undeformed) two-point function of $\mathcal{O}$,

$$
(\hat{G} \sigma)(x)=\int d^{d} z\langle\mathcal{O}(x) \mathcal{O}(z)\rangle_{0} \sigma(z)=\int d^{d} z \frac{\sigma(z)}{|x-z|^{2 \Delta_{-}}},
$$

and the operators

$$
\hat{K}_{f}=1+f \hat{G}, \quad \hat{Q}_{f}=\frac{\hat{G}}{1+f \hat{G}} .
$$

Clearly these kernels become diagonal in momentum space,

$$
G(k)=\int \frac{d^{d} k}{(2 \pi)^{d}} \frac{e^{i k x}}{x^{2 \Delta_{-}}}=A_{\nu} k^{-2 \nu}, \quad A_{\nu} \equiv 2^{2 \nu} \pi^{d / 2} \frac{\Gamma(\nu)}{\Gamma\left(\frac{d}{2}+\nu\right)} .
$$

and

$$
K_{f}=1+f A_{\nu} k^{-2 \nu}, \quad Q_{f}=\frac{A_{\nu} k^{-2 \nu}}{1+f A_{\nu} k^{-2 \nu}} .
$$

With these notations in place, the path integral over $\sigma$ gives

$$
Z_{f}[J]=\frac{1}{\sqrt{\operatorname{det} \hat{K}_{f}}} \exp \left(\int J \hat{Q}_{f} J\right) .
$$

Hence in the presence of the deformation, the two point function is

$$
\left\langle\mathcal{O}\left(x_{1}\right) \mathcal{O}\left(x_{2}\right)\right\rangle_{f}=\left.\frac{\delta^{2} \log Z_{f}[J]}{\delta J\left(x_{1}\right) \delta J\left(x_{2}\right)}\right|_{J=0}=Q_{f}\left(x_{1}, x_{2}\right) .
$$

In the IR, where $f G \gg 1$, we have

$$
Q_{f}(k)=\frac{1}{f}-\frac{1}{f^{2} G(k)}+\cdots .
$$


In position space $Q_{f=\infty}(x, 0) \sim 1 / x^{2 \Delta_{+}}$, with $\Delta_{+} \equiv \frac{d}{2}+\nu$. The double-trace deformation triggers an $\mathrm{RG}$ flow that leads to a new IR fixed point; the dimension of $\mathcal{O}$ flows from $\Delta_{-}$in the UV to $\Delta_{+}$in the IR. A simple manipulation of the generating functional (2.3, 2.4) shows that the UV and IR large $N$ CFTs are related by a Legendre transform 3 .

Still following [3], to determine the central charge we can place the CFT on a $d$-sphere of radius $R$ and compute

$$
c \equiv\left\langle\int_{S_{R}^{d}} d^{d} x \sqrt{g} T_{\mu}^{\mu}\right\rangle=\frac{1}{d} R \frac{\partial}{\partial R} W[R], \quad W[R] \equiv \log Z\left[J=0, S_{R}^{d}\right] .
$$

(Note that for $d=4$, this definition coincides - up to a universal numerical factor - with what is usually called the " $a$ " anomaly coefficient - the coefficient in front of the Euler density in the expression for the trace anomaly $T_{\mu}^{\mu}$ in a curved background. Indeed the other curvature invariant is the Weyl tensor, which vanishes on the conformally flat $\mathbf{S}_{R}^{d}$.) As usual in quantum field theory, the partition function is UV divergent. Introducing a UV (momentum) cutoff $\Lambda$, by scale invariance we must have $W=f(\Lambda R)=\alpha_{d}(\Lambda R)^{d}+\alpha_{d-1}(\Lambda R)^{d-1}+\cdots \alpha_{0} \log (\Lambda R)+\cdots$. Equation (2.12) must be interpreted as valid for the renormalized $W[R]$ - in practice this means that we focus on the logarithmic divergence, so that $c \equiv \alpha_{0} / d$. As is well-known, a non-zero anomaly can arise only for even $d$.

We are interested in finding

$$
c_{I R}-c_{U V}=\frac{1}{d} R \frac{\partial}{\partial R}\left(W_{f=\infty}[R]-W_{f=0}[R]\right) .
$$

From the analysis with the auxiliary field method,

$$
W_{f_{1}}[R]-W_{f_{2}}[R]=-\frac{1}{2} \operatorname{tr} \log \left(\frac{1+f_{1} \hat{G}}{1+f_{2} \hat{G}}\right)=-\frac{1}{2} \operatorname{tr} \log \left(\frac{\hat{Q}_{f_{2}}}{\hat{Q}_{f_{1}}}\right) .
$$

The eigenvalues $g_{l}$ of $\hat{G}$ on $\mathbf{S}_{R}^{d}$ are calculated using an expansion in spherical harmonics,

$$
G\left(x, x^{\prime}\right)=\left\langle\mathcal{O}(x) \mathcal{O}\left(x^{\prime}\right)\right\rangle_{0}=\sum_{l, m} g_{l} Y_{l m}^{*}(x) Y_{l m}\left(x^{\prime}\right),
$$

which gives 3 .

$$
g_{l}=R^{2 \nu} \pi^{d / 2} 2^{2 \nu} \frac{\Gamma(\nu) \Gamma\left(l+\frac{d}{2}-\nu\right)}{\Gamma\left(\frac{d}{2}-\nu\right) \Gamma\left(l+\frac{d}{2}+\nu\right)} .
$$

Plugging this into (2.14), a fair amount of work still needs to be done to extract the logarithmic divergence $\sim \log (\Lambda R)$ and find the change in the central charge. 
The sum can be calculated [3] with a zeta function regulator, which automatically gets rid of the power law divergences in $W$. In the following, we will directly compare the change in the CFT partition function (2.14) with the corresponding quantity in AdS space, so we will not need to use anything beyond equations (2.14,2.16).

\section{Bulk: mixed boundary conditions}

If the unperturbed CFT has a dual description as a gravitational theory in $\operatorname{AdS}_{d+1}$, then its deformation by $\frac{f}{2} \mathcal{O}^{2}$ has a simple holographic interpretation [1].

Let us recall some basic facts about the AdS/CFT correspondence. We write the Euclidean AdS metric as (the AdS scale is set to one)

$$
d s^{2}=\frac{1}{r^{2}}\left(d r^{2}+A(r) g_{i j}(x) d x^{i} d x^{j}\right)
$$

where $x^{i}, i=1, \cdots d$, parametrize the conformal boundary at $r=0$ and $A(r) \rightarrow 1$ as $r \rightarrow 0$. Popular choices are Poincaré coordinates $r \equiv x_{0}, 0<x_{0}<\infty, A=1$, $g_{i j}=\delta_{i j}$, for which the boundary is flat $\mathbf{R}^{d}$, and hyperbolic coordinates $r \equiv \rho$, $0<\rho \leq 2, A(\rho)=\left(\frac{4-\rho^{2}}{4}\right)^{2}, g_{i j} d x^{i} d x^{j}=d \Omega_{d}$, for which the boundary is $\mathbf{S}^{d}$. For a free scalar field of mass $m$, the solution to the wave equation near the boundary $r \rightarrow 0$ takes the form

$$
\phi(x, r)=r^{\Delta_{+}}\left[\alpha(x)+O\left(r^{2}\right)\right]+r^{\Delta_{-}}\left[\beta(x)+O\left(r^{2}\right)\right],
$$

where we have defined

$$
\Delta_{ \pm}=\frac{d}{2} \pm \nu, \quad \nu=\sqrt{\frac{d^{2}}{4}+m^{2}}
$$

The Breitenlohner-Freedman bound [24] $m^{2} \geq-\frac{d^{2}}{4}$ is necessary for stability and ensures that $\Delta_{ \pm}$are real. For general masses, the field must be quantized with the boundary condition $\beta=0$, the so-called "regular" choice of boundary conditions. In the range $-\frac{d^{2}}{4} \leq m^{2}<-\frac{d^{2}}{4}+1$ (equivalently $0 \leq \nu<1$ ), the "irregular" choice $\alpha=0$ is also possible. This can be understood (in Lorentzian signature) from the fact that regular solutions are always normalizable, while irregular solutions are normalizable only in this restricted range of masses. With the regular choice of boundary conditions, we identify $\beta(x)$ with the source for an operator $\mathcal{O}$ in the boundary CFT - the CFT action has a term $\int d^{d} x \beta(x) \mathcal{O}(x)$. Under the scaling isometry $r \rightarrow \lambda r, \phi$ is invariant hence $\mathcal{O}$ has dimension $d-\Delta_{-}=\Delta_{+}$. The function $\alpha$ is identified with the expectation value of the operator, $\alpha=\langle\mathcal{O}\rangle$. The irregular choice of boundary conditions corresponds instead to identifying $\alpha(x)$ as 
the source of the boundary operator and $\beta$ as the vev [23]; then $\mathcal{O}$ has dimension $\Delta_{-}$. In going from the regular to the irregular boundary conditions, the roles of the source and of the vev are reversed, and the generating functions of correlation functions of the two theories are related by a Legendre transformation [23]. In section 5 we shall give a direct proof of this fact.

In fact in the mass range $-\frac{d^{2}}{4} \leq m^{2}<-\frac{d^{2}}{4}+1$, a large class of boundary conditions are possible, of the form $\alpha(x)=F[\beta(x)]$, where $F$ is any real functional. For generic $F$ the AdS isometry group is not preserved. Witten [1] has interpreted these general boundary conditions as a multi-trace deformation of the boundary CFT, of the form $\int d^{d} x \mathcal{W}[\mathcal{O}(x)]$, with $F[\beta]=\frac{\delta \mathcal{W}}{\delta \beta}$. In the case of a double-trace deformation, $\mathcal{W}(\mathcal{O})=\frac{f}{2} \mathcal{O}^{2}$, which leads to the boundary conditions ${ }^{2}$

$$
\alpha(x)-\tilde{f} \beta(x)=0
$$

This dovetails perfectly with the field theoretic analysis of the previous section. In the UV, $f \rightarrow 0$ and the field is quantized with irregular boundary conditions, so that the dual operator $\mathcal{O}$ has $\Delta_{-}$dimension; in the IR $f \rightarrow \infty$ the regular boundary conditions are approached and $\mathcal{O}$ has dimension $\Delta_{+}$.

\subsection{A regulator}

To make precise sense of the AdS/CFT prescription it is often necessary (see e.g. [25, 26]) to introduce an IR bulk regulator - which by the UV/IR connection plays the role of a boundary UV regulator. We cut off the infinite volume of AdS space by restricting the radial coordinate to $r \geq \epsilon>0$, and setup the boundary value problem on the surface $r=\epsilon$.

Let us see how to write the boundary conditions (3.4) in the presence of this cutoff. ${ }^{3}$ The action for a scalar field is

$$
S=\int d^{d} x d r \sqrt{g}\left(\frac{1}{2}(\partial \phi)^{2}+\frac{1}{2} m^{2} \phi^{2}\right)+\left.\int d^{d} x \sqrt{g} \mathcal{L}_{b d r y}\right|_{r=\epsilon} .
$$

Varying the field, we find upon integrating by parts and using the bulk equations of motion,

$$
\delta S=\int d^{d} x \sqrt{g}\left[\delta \phi \partial \phi \cdot \hat{n}+\delta \mathcal{L}_{b d r y}\right]_{r=\epsilon},
$$

\footnotetext{
${ }^{2}$ Here $\tilde{f}=$ const $\cdot f$ where the proportionality constant depends on the conventional normalization of $\mathcal{O}$ and will be determined below. In section 2, we normalized $\mathcal{O}$ to have unit two-point function in the unperturbed theory, see (2.5). This normalization differs from the one obtained by taking functional derivatives with respect to the source $\alpha(x)$, which is the normalization implicit in Witten's prescription. This is why $f \rightarrow \tilde{f}$ in (3.4).

${ }^{3}$ See also the closely related discussion in [5, 9].
} 
where $\hat{n}=\epsilon \hat{r}$ is the unit vector specifying the normal to the boundary. In order for the variational problem to be well-defined, we must choose the boundary condition for the field to cancel the contribution of the boundary term in the action. To reproduce the deformation $\frac{f}{2} \mathcal{O}^{2}$ added to the CFT, we choose the boundary term

$$
\mathcal{L}_{b d r y}=\frac{1}{2} \gamma \phi^{2}
$$

which dictates the mixed Neumann/Dirichlet boundary conditions ${ }^{4}$

$$
\gamma \phi(x, \epsilon)+\partial \phi(x, \epsilon) \cdot \hat{n}=0 \text {. }
$$

Now we must relate $f$ to $\gamma$. To this end, we are going to compute the two-point function using the AdS/CFT prescription and compare it with the field theoretic result of the previous section. In $\mathrm{AdS} / \mathrm{CFT}$, we are instructed to evaluate the on-shell bulk action as a functional of the boundary source $\phi_{b}(x)$ [27, 28]. For our mixed boundary conditions, the appropriate boundary value problem is

$$
\begin{aligned}
\left(\square-m^{2}\right) \phi(x, r) & =0 \\
\gamma \phi(x, \epsilon)+\partial \phi(x, \epsilon) \cdot \hat{n} & =\phi_{b}(x) .
\end{aligned}
$$

Plugging the solution of (3.9) back into the action and integrating by parts,

$$
\begin{aligned}
S_{\text {on-shell }}\left[\phi_{b}\right] & =\frac{1}{2} \int d^{d} x \sqrt{g}\left[\left.\phi(x, r)(\partial \phi(x, r) \cdot \hat{n}+\gamma \phi(x, r)]\right|_{r=\epsilon}\right. \\
& =\frac{1}{2} \int d^{d} x \sqrt{g} \phi(x, \epsilon) \phi_{b}(x) .
\end{aligned}
$$

To obtain an explicit solution, let us specialize to Poincaré coordinates,

$$
d s^{2}=\frac{1}{x_{0}^{2}}\left(d x_{0}^{2}+d x_{i} d x_{i}\right), \quad x_{0} \geq \epsilon,
$$

and Fourier transform with respect to the flat boundary coordinates, $x_{i} \rightarrow k_{i}$, $i=1, \cdots d$,

$$
\phi\left(x_{i}, x_{0}\right)=\frac{1}{(2 \pi)^{d / 2}} \int d^{d} k e^{i k_{i} x_{i}} \phi\left(k_{i}, x_{0}\right) .
$$

The wave equation reads

$$
\left[-x_{0}^{d+1} \frac{\partial}{\partial x_{0}}\left(x_{0}^{-d+1} \frac{\partial}{\partial x_{0}}\right)+k^{2} x_{0}^{2}+m^{2}\right] \phi\left(k, x_{0}\right)=0
$$

\footnotetext{
${ }^{4}$ For all $\gamma$, Dirichlet conditions $\delta \phi=0$ are also consistent, but we choose to impose (3.8).
} 
with $k=\sqrt{k_{i} k_{i}}$. As is well-known (see e.g. [27, 25]), the unique solution that is regular for $x_{0} \rightarrow \infty$ is

$$
\psi\left(k, x_{0}\right) \equiv x_{0}^{d / 2} \mathcal{K}_{\nu}\left(k x_{0}\right),
$$

where $\mathcal{K}_{\nu}$ is the Bessel function. Thus the solution of the boundary value problem (3.9) is

$$
\phi(k, r)=\left(\frac{\psi(k, r)}{\gamma \psi(k, \epsilon)+\partial \psi(k, \epsilon) \cdot \hat{n}}\right) \phi_{b}(k)
$$

and the AdS/CFT dictionary yields the CFT correlator

$$
\begin{aligned}
\left\langle\mathcal{O}(k) \mathcal{O}\left(k^{\prime}\right)\right\rangle & =-\left.\frac{\delta^{2} S_{\text {on-shell }}\left[\phi_{b}\right]}{\delta \phi_{b}(k) \delta \phi_{b}\left(k^{\prime}\right)}\right|_{\phi_{b}=0} \\
& =-\epsilon^{-d} \delta^{d}\left(k+k^{\prime}\right)\left(\frac{\psi(k, \epsilon)}{\gamma \psi(k, \epsilon)+\partial \psi(k, \epsilon) \cdot \hat{n}}\right) .
\end{aligned}
$$

Using the expansion of $\mathcal{K}_{\nu}(z)=z^{-\nu}\left[2^{-1+\nu} \Gamma(\nu)+O\left(z^{2}\right)\right]+z^{\nu}\left[2^{-1-\nu} \Gamma(-\nu)+O\left(z^{2}\right)\right]$, we can extract the leading behavior of the two point function as $\epsilon \rightarrow 0$,

$$
\left\langle\mathcal{O}(k) \mathcal{O}\left(k^{\prime}\right)\right\rangle_{\gamma}=-\frac{\epsilon^{-d} \delta^{d}\left(k+k^{\prime}\right)}{\gamma+\Delta_{-}+\left(\epsilon^{2 \nu} 2^{-2 \nu} \frac{\Gamma(-\nu)}{\Gamma(\nu)}(2 \nu)\right) k^{2 \nu}+O\left(\epsilon^{2}\right)} .
$$

Notice that in the mass range that we are considering, $\nu<1$ and the term $O\left(\epsilon^{2}\right)$ can indeed be neglected. On the other hand, the two-point function was computed directly in the boundary CFT using the auxiliary field method,

$$
\left\langle\mathcal{O}(k) \mathcal{O}\left(k^{\prime}\right)\right\rangle_{f}=\delta^{d}\left(k+k^{\prime}\right) Q_{f}(k)=\delta^{d}\left(k+k^{\prime}\right) \frac{A_{\nu}}{f A_{\nu}+k^{2 \nu}} .
$$

The two expressions coincide (up to an overall $k$-independent normalization that can be fixed by rescaling the source $\phi_{b}(x)$ ), provided we identify

$$
\gamma=-\Delta_{-}-f \epsilon^{2 \nu}\left(2 \pi^{d / 2} \frac{\Gamma(1-\nu)}{\Gamma\left(\Delta_{-}\right)}\right)
$$

As $f \rightarrow \infty$, we find that $\gamma \rightarrow \infty$, and recover the Dirichlet boundary value problem at $x_{0}=\epsilon$, which is indeed the usual prescription for the "regular" $\Delta_{+}$ quantization. We see that the "irregular" $\Delta_{-}$quantization $(f=0)$ corresponds to the specific choice $\gamma=-\Delta_{-}$in the boundary conditions (3.4). This can be understood by the following simple argument. The irregular boundary conditions consists of identifying the subleading term $\alpha(x)$ in the boundary expansion of $\phi$ as the source for $\mathcal{O}$. Now the boundary source at $r=\epsilon$ is

$$
\begin{aligned}
\phi_{b}(x) & =\gamma \phi(x, \epsilon)+\left.\epsilon \partial_{r} \phi(x, r)\right|_{r=\epsilon} \\
& =\epsilon^{\Delta_{-}}\left[\left(\gamma+\Delta_{-}\right) \beta(x)+O\left(\epsilon^{2}\right)\right]+\epsilon^{\Delta_{+}}\left[\left(\gamma+\Delta_{+}\right) \alpha(x)+O\left(\epsilon^{2}\right)\right],
\end{aligned}
$$


and indeed to cancel the dependence from the leading term $\beta$ we must choose $\gamma=-\Delta_{-} \cdot{ }^{5}$ In summary, the double-trace deformation $\int \frac{f}{2} \mathcal{O}^{2}$ of the boundary CFT corresponds to imposing mixed Neumann/Dirichlet boundary conditions (3.8) for the dual scalar field $\phi$, with $\gamma$ and $f$ related by (3.19). These boundary conditions become more transparent in terms of the field $\chi$ introduced in [23], locally related to $\phi$ as

$$
\phi\left(x_{i}, x_{0}\right) \equiv x_{0}^{\Delta_{-}} \chi\left(x_{i}, x_{0}\right) .
$$

Then $\Delta_{-}$quantization corresponds precisely to Neumann boundary conditions for $\chi$,

$$
\frac{\partial}{\partial x_{0}} \chi=0
$$

while, of course, $\Delta_{+}$quantization corresponds to Dirichlet conditions.

General multitrace deformations $\int d^{d} x \mathcal{W}[\mathcal{O}(x)]$ could be treated similarly, by taking $\mathcal{L}_{\text {bdry }}=\mathcal{W}(\phi)$.

\section{Relating the bulk and boundary partition functions}

We are now going to show that the change in the partition function $W$ induced by the double trace deformation is manifestly identical on both sides of the correspondence. In AdS, we look at the variation of the partition function for a scalar field of mass $m$ as we change boundary conditions,

$$
W_{\gamma_{1}}^{A d S}-W_{\gamma_{2}}^{A d S}=-\frac{1}{2} \operatorname{Tr}_{\gamma_{1}} \log \left(-\square+m^{2}\right)+\frac{1}{2} \operatorname{Tr}_{\gamma_{2}} \log \left(-\square+m^{2}\right)
$$

The trace " $\operatorname{Tr}_{\gamma}$ " is over the bulk modes that obey the boundary conditions (3.19). Separating the AdS coordinates into a radial coordinate and $d$ coordinates parametrizing the boundary, we can also write

$$
W_{\gamma_{1}}^{A d S}-W_{\gamma_{2}}^{A d S}=-\frac{1}{2} \operatorname{tr} \log \left(\frac{\operatorname{det}_{\gamma_{1}}\left(-\square+m^{2}\right)}{\operatorname{det}_{\gamma_{2}}\left(-\square+m^{2}\right)}\right),
$$

where the trace "tr" is over the boundary modes only, and the determinant $\operatorname{det}_{\gamma}$ is the product of eigenvalues of the radial wave-equation with $\gamma$ boundary conditions. Here " $\left(-\square+m^{2}\right)$ " is a short-hand for the radial differential operator, which depends on the eigenvalue of the boundary Laplacian. For example, in

\footnotetext{
${ }^{5}$ Incidentally, we can also determine the relation between $f$ and the parameter $\tilde{f}$ introduced in (3.4). Substituting (3.19) in (3.20) and setting $\phi_{b}=0, \alpha=\tilde{f} \beta$, we find $\tilde{f}=\frac{\pi^{d / 2} \Gamma(1-\nu)}{\nu \Gamma\left(\Delta_{-}\right)} f$, in agreement with $\underline{3}$. We will not need this relation in the following.
} 
Poincaré coordinates, it is the second-order differential operator in $x_{0}$ given in (3.13), which depends on the boundary momentum $k$.

The corresponding quantity in the dual CFT is (2.14)

$$
W_{f_{1}}^{C F T}-W_{f_{2}}^{C F T}=-\frac{1}{2} \operatorname{tr} \log \left(\frac{1+f_{1} G}{1+f_{2} G}\right) .
$$

Equating the change in the central charge on both sides of the AdS/CFT correspondence thereby reduces to confirming that

$$
\frac{\operatorname{det}_{\gamma_{1}}\left(-\square+m^{2}\right)}{\operatorname{det}_{\gamma_{2}}\left(-\square+m^{2}\right)}=\frac{1+f_{1} G}{1+f_{2} G}=\frac{Q_{f_{2}}}{Q_{f_{1}}} .
$$

This will be our task for the rest of the section.

\subsection{One-dimensional determinants}

Fortunately, the ratio of one-dimensional determinants that appears in (4.4) belongs to a well studied class of problems. As a paradigmatic example, consider the two differential operators in one dimension

$$
\begin{aligned}
\mathcal{Q} & =-\frac{d^{2}}{d x^{2}}+R_{\mathcal{Q}}(x) \\
\mathcal{P} & =-\frac{d^{2}}{d x^{2}}+R_{\mathcal{P}}(x) .
\end{aligned}
$$

The problem is to find the ratio of functional determinants

$$
\frac{\operatorname{det}(\mathcal{Q}-\lambda)}{\operatorname{det}(\mathcal{P}-\lambda)}
$$

in the space of (square-integrable) functions $\mathcal{F}=\{f(x)\}, x \in[a, b]$, that satisfy specific boundary conditions - for simplicity let us say Dirichlet boundary conditions $f(a)=f(b)=0$. This problem has an elegant solution. Define the functions $q_{\lambda}$ and $p_{\lambda}$ to be eigenfunctions of the two operators,

$$
\begin{aligned}
& \mathcal{Q} q_{\lambda}=\lambda q_{\lambda} \\
& \mathcal{P} p_{\lambda}=\lambda p_{\lambda}
\end{aligned}
$$

with the boundary conditions

$$
\begin{array}{ll}
q(b)=0 & q^{\prime}(b)=1 \\
p(b)=0 & p^{\prime}(b)=1 .
\end{array}
$$


Notice that in general $q_{\lambda}$ and $p_{\lambda}$ do not belong to $\mathcal{F}$. Then

$$
\frac{\operatorname{det}(\mathcal{Q}-\lambda)}{\operatorname{det}(\mathcal{P}-\lambda)}=\frac{q_{\lambda}(a)}{p_{\lambda}(a)} .
$$

In his famous lectures on instantons [29, Coleman gives the following heuristic proof of this equation. Viewed as complex functions of $\lambda$, both sides of the equation have the same poles and zeros. This is the case because for all $\lambda, q_{\lambda}$ satisfies one of the boundary conditions, $q(b)=0$; if it also satisfies the other boundary condition $q_{\lambda}(a)=0$, then $q_{\lambda}$ is an eigenfunction of $\mathcal{Q}$ in $\mathcal{F}$ and thus $\mathcal{Q}-\lambda$ has vanishing determinant. The same applies to $p$ and $\mathcal{P}$. Both sides of the equation also have the same limiting behavior as $\lambda \rightarrow \infty$, so they must in fact be the same function.

In [30], a rigorous proof is given of a more general theorem. Formulas analogous to (4.10) allow us to compute ratios of functional determinants for general Sturm-Liouville operators of the type

$$
\begin{aligned}
\mathcal{Q} & =-\frac{d}{d x}\left(S_{\mathcal{Q}}(x) \frac{d}{d x}\right)+R_{\mathcal{Q}}(x) \\
\mathcal{P} & =-\frac{d}{d x}\left(S_{\mathcal{P}}(x) \frac{d}{d x}\right)+R_{\mathcal{P}}(x),
\end{aligned}
$$

acting on the space of functions with some prescribed mixed Neumann/Dirichlet boundary conditions at the extrema. Consider for definiteness the space of functions $\mathcal{F}_{\gamma}$ with Dirichlet conditions at $x=b$ and mixed boundary conditions at $x=a$,

$$
\begin{aligned}
f(a) & =0 \\
\gamma f(a)+f^{\prime}(a) & =0 .
\end{aligned}
$$

Since the boundary conditions are parametrized by $\gamma$, we will denote the resulting determinant by $\operatorname{det}_{\gamma}$. If $q_{\lambda}$ and $p_{\lambda}$ are defined again as in (4.8, 4.9), then provided that $S_{\mathcal{Q}}=S_{\mathcal{P}}$ one has 30 .

$$
\frac{\operatorname{det}_{\gamma} \mathcal{Q}}{\operatorname{det}_{\gamma} \mathcal{P}}=\frac{\gamma q(a)+q^{\prime}(a)}{\gamma p(a)+p^{\prime}(a)},
$$

where we have relabelled $q \equiv q_{\lambda=0}, p \equiv p_{\lambda=0}$.

One final complication is that in this formula, the same choice of $\gamma$ must appear in both determinants, while the two operators $\mathcal{P}$ and $\mathcal{Q}$ are different. To check equation (4.4), we need to compute a ratio of determinants where the 
operators are the same but the boundary conditions are different. The obvious guess in this case is

$$
\frac{\operatorname{det}_{\gamma_{1}} \mathcal{P}}{\operatorname{det}_{\gamma_{2}} \mathcal{P}}=\frac{\gamma_{1} p(a)+p^{\prime}(a)}{\gamma_{2} p(a)+p^{\prime}(a)}
$$

which has the correct poles and zeros. We will prove this formula by generalizing the proof given in [30]. The zeta function of the differential operator $\mathcal{P}$ is defined as usual,

$$
\zeta_{\mathcal{P}_{\gamma}}(s)=\sum_{\lambda} \lambda^{-s}
$$

where the sum is over eigenvalues of $\mathcal{P}$, and we have added a label $\gamma$ to denote the boundary condition explicitly. Knowledge of the $\zeta$ function allows of course to compute the determinant, since $\zeta_{\mathcal{P}}^{\prime}(0)=-\log \operatorname{det} \mathcal{P}$. The basic observation is that we can write

$$
\zeta_{\mathcal{P}_{\gamma_{1}}}(s)-\zeta_{\mathcal{P}_{\gamma_{2}}}(s)=\frac{1}{2 \pi i} \int d \lambda \lambda^{-s} \frac{d}{d \lambda} \log \frac{\gamma_{1} p_{\lambda}(a)+p_{\lambda}^{\prime}(a)}{\gamma_{2} p_{\lambda}(a)+p_{\lambda}^{\prime}(a)} .
$$

Indeed $\lambda$ is an eigenvalue of $\mathcal{P}_{\gamma}$ if and only if the function $\gamma p_{\lambda}(a)+p^{\prime}(\lambda)$ has a zero; correspondingly its logarithmic derivative $\frac{d}{d \lambda} \log \left[\gamma p_{\lambda}(a)+p_{\lambda}^{\prime}(a)\right]$ has a pole with unit residue and the contour integration in the complex $\lambda$ plane reproduces the definition (4.14). The contour of integration is shown in Fig. 11. The branch cut for $\lambda^{-s}$ is placed at an angle $\theta$ from the positive real $\lambda$ axis. As it stands, this definition is meaningless at $s=0$. The goal is to deform the contour to enclose the branch cut, and obtain an expression that extends to a region around $s=0$. If the integrand behaves as

$$
\frac{d}{d \lambda} \log \frac{\gamma_{1} p_{\lambda}(a)+p_{\lambda}^{\prime}(a)}{\gamma_{2} p_{\lambda}(a)+p_{\lambda}^{\prime}(a)}=\mathcal{O}\left(\frac{1}{\lambda^{3 / 2}}\right)
$$

as $\Im \sqrt{\lambda} \rightarrow \pm \infty$, then the contour can be deformed and the resulting integral is well defined for $-1 / 2<s<1$. Differentiating (4.15) with respect to $s$ and setting $s=0$ gives exactly (4.13), completing the proof. (See [30] for a careful analysis of the case (4.12)). The assumption (4.16) can be checked for any particular case; we will show below that it holds in our case. ${ }^{6}$

\subsection{Relating the partition functions}

We are now in a position to verify equation (4.4). We apply the formula (4.13) with $\mathcal{P}=$ " $\left(-\square+m^{2}\right)$ " the radial differential operator. The left extremum is

\footnotetext{
${ }^{6}$ In [30, the analogous asymptotic behavior for the case (4.12) was argued to hold for general Sturm-Liouville operators $\mathcal{Q}$ and $\mathcal{P}$. In our case, although $\mathcal{P}$ is not of Sturm-Liouville type, we will be able to check 4.16] explicitly.
} 


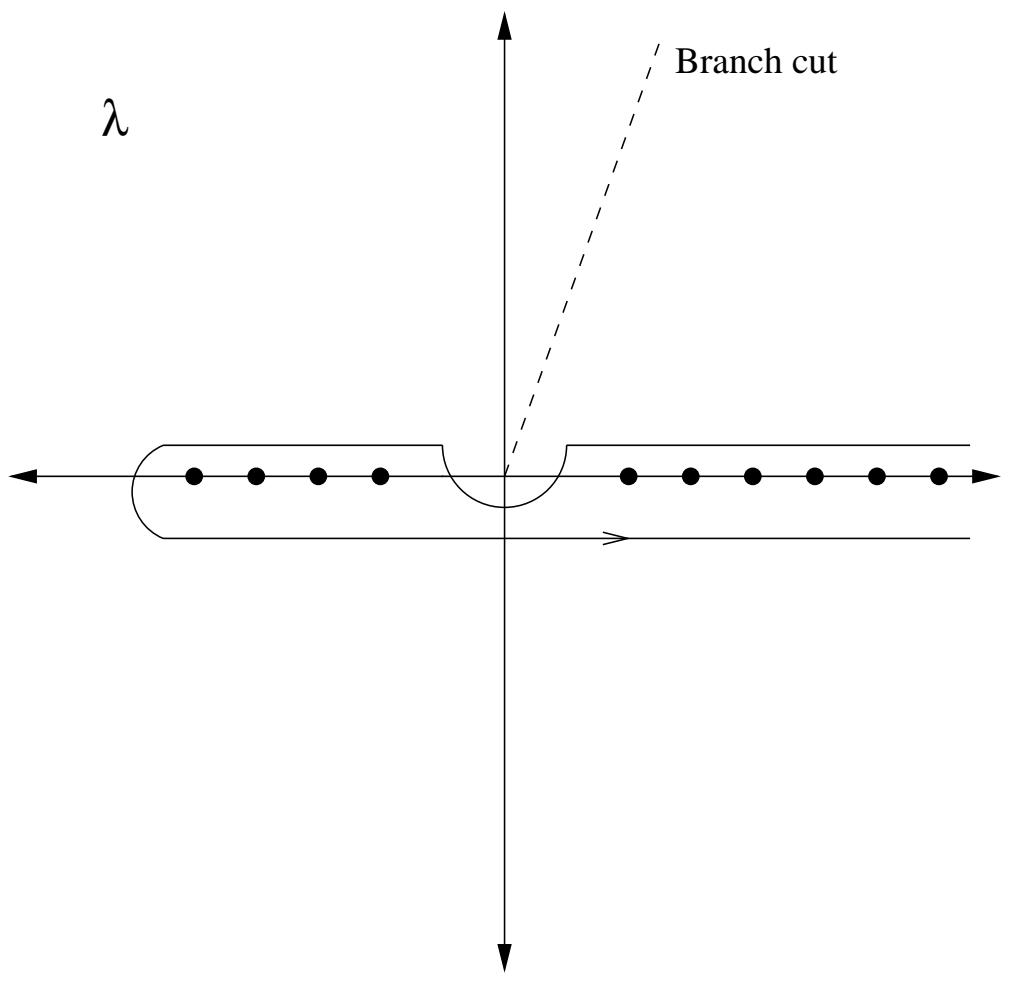

Figure 1: The contour and branch cut in Eq. [4.15] [30].

taken $r=a=\epsilon$, while the right extremum is taken to correspond to the "deep interior" of AdS - for example $x_{0}=\infty$ in Poincaré coordinates and $\rho=2$ in hyperbolic coordinates. We immediately have

$$
\frac{\operatorname{det}_{\gamma_{1}}\left(-\square+m^{2}\right)}{\operatorname{det}_{\gamma_{2}}\left(-\square+m^{2}\right)}=\frac{\gamma_{1} p(\epsilon)+\epsilon p^{\prime}(\epsilon)}{\gamma_{2} p(\epsilon)+\epsilon p^{\prime}(\epsilon)},
$$

where $p$ is the solution of the radial wave equation obeying Dirichlet boundary conditions in the interior of AdS. For example in Poincaré coordinates, $p\left(k, x_{0}\right)=$ $\psi\left(k, x_{0}\right)$ as defined in (3.14). Using the identity

$$
\frac{p(\epsilon)}{\gamma p(\epsilon)+\epsilon p^{\prime}(\epsilon)}=Q_{f}
$$


which was shown to hold in section 3 (see (13.16) and below ${ }^{7}$ ), we have finally

$$
\frac{\operatorname{det}_{\gamma_{1}}\left(-\square+m^{2}\right)}{\operatorname{det}_{\gamma_{2}}\left(-\square+m^{2}\right)}=\frac{Q_{f_{2}}}{Q_{f_{1}}}
$$

It follows that the change in the partition function of the AdS theory is identical to that of the CFT, term by term in a sum over boundary modes.

\subsection{Explicit Analysis: Poincaré Coordinates}

To clarify the discussion above in generic coordinates, we will demonstrate the construction explicitly in Poincaré coordinates,

$$
d s^{2}=\frac{1}{x_{0}^{2}}\left(d x_{0}^{2}+d x^{2}\right)
$$

The change in the CFT effective potential from equation (2.14) is

$$
\Delta V^{C F T}=-\frac{1}{2} \int \frac{d^{d} k}{(2 \pi)^{d}} \log \left(1+f g_{k}\right)
$$

where $g_{k}$ is the eigenvalue of the CFT propagator. Using

$$
\begin{aligned}
\left\langle\mathcal{O}_{\Delta}(x) \mathcal{O}_{\Delta}(y)\right\rangle & =\frac{1}{|x-y|^{2 \Delta}} \\
& =\int \frac{d^{d} k}{(2 \pi)^{d}} e^{-i k(x-y)} g_{k}
\end{aligned}
$$

we find

$$
g_{k}=k^{-2 \nu} \pi^{d / 2} \frac{2^{2 \nu}}{\nu} \frac{\Gamma(1+\nu)}{\Gamma(d / 2-\nu)} .
$$

The change in the AdS effective potential as we switch from irregular to regular modes is given by equation (4.2) with $\gamma_{1} \rightarrow \infty, \gamma_{2}=-\Delta_{-}$.

To evaluate the ratio of determinants using eq. 4.13, we must first analyze the large $\nu$ behavior of

$$
\frac{\gamma_{1} q_{\lambda}(a)+\epsilon q_{\lambda}^{\prime}(a)}{\gamma_{2} q_{\lambda}(a)+\epsilon q_{\lambda}^{\prime}(a)}
$$

with $q_{\lambda}(y)=y^{d / 2} \mathcal{K}_{\nu}(k y)$. (In this case, the roots in the integrand of the zeta function lie on the imaginary axis, so we must check that the large $\Re \nu$ behavior

\footnotetext{
${ }^{7}$ The argument below (3.16) was phrased for definiteness in Poincaré coordinates, but the conclusion is clearly general.
} 
is no worse than $\mathcal{O}\left(\lambda^{-3 / 2}\right)$.) Using the fact that $\mathcal{K}_{\nu+1}(x) / \mathcal{K}_{\nu}(x) \rightarrow 2 \nu / x$ as $\nu \rightarrow \infty$, we find

$$
\frac{d}{d \lambda} \log \frac{\gamma_{1} q_{\lambda}(a)+\epsilon q_{\lambda}^{\prime}(a)}{\gamma_{2} q_{\lambda}(a)+\epsilon q_{\lambda}^{\prime}(a)}=\frac{a\left(\gamma_{1}-\gamma_{2}\right)}{\epsilon}\left(\frac{1}{\nu^{2}}\right)+\mathcal{O}\left(\frac{1}{\nu^{3}}\right)
$$

This confirms assumption (4.16). Finally, plugging into eq. (4.13) and substituting $f$ for $\gamma$, we find precisely the answer we found for $\Delta V^{C F T}$ in equation (4.21). This completes the demonstration in Poincar'e coordinates that

$$
\Delta V^{A d S}=\Delta V^{C F T} .
$$

\subsection{Explicit analysis: Hyperbolic Coordinates}

To make contact with the results of [2] and [3], we now perform an explicit analysis in hyperbolic coordinates, where the boundary of AdS is a sphere. The metric is

$$
d s^{2}=\frac{d \rho^{2}}{\rho^{2}}+\left(\frac{4-\rho^{2}}{4 \rho}\right)^{2} d \Omega_{d}^{2} .
$$

Expanding in spherical harmonics

$$
\phi(\rho, \Omega)=\sum_{l, m} Y_{l m}(\Omega) \phi_{l}(\rho)
$$

the radial wave equation reads

$$
\left[\rho^{2} \frac{\partial}{\partial \rho^{2}}+\rho \frac{\partial}{\partial \rho}+d \rho \frac{\rho^{2}+4}{\rho^{2}-4} \frac{\partial}{\partial \rho}-l(l+d-1)\left(\frac{4 \rho}{4-\rho^{2}}\right)^{2}\right] \phi_{l}(\rho)=0 .
$$

The unique solution regular in the "deep interior" at $\rho=2$ is

$$
p_{l}(\rho)=\left(\frac{4-\rho^{2}}{4 \rho}\right)^{l} F\left(l+\Delta_{+}, l+\Delta_{-}, l+\frac{d}{2}+\frac{1}{2} ;-\frac{(\rho-2)^{2}}{8 \rho}\right) .
$$

Notice that $p_{l}$ is zero at $\rho=2$ together with its first $l-1$ derivatives. The boundary conditions at the right extremum $b=2$ are taken to be

$$
p_{l}(b)=0, \quad \frac{d^{l} p_{l}(b)}{d \rho^{l}}=\frac{l !}{(-2)^{l}} .
$$

This is an inessential modification of the condition (4.9).

Expanding near the boundary,

$$
p_{l}(\epsilon) \approx a_{l} \epsilon^{\Delta_{+}}+b_{l} \epsilon^{\Delta_{-}},
$$




\begin{tabular}{|l|c|c|}
\hline & $R_{\text {numerical }}$ & $R_{\text {analytical }}$ \\
\hline Point A: $\gamma_{1}=1, \gamma_{2}=10$ & -0.0336349 & -0.0336350 \\
\hline Point B: $\gamma_{1}=10, \gamma_{2}=100$ & 0.0882113 & 0.0882118 \\
\hline
\end{tabular}

Table 1: Comparison of numerical and analytical computations of (4.37). The analytical results were computed using eq. (4.13). The numerical results were found by computing approximately 30000 eigenvalues for each operator and extrapolating to $n \rightarrow \infty$. In all cases, $d=4, l=3, \epsilon=10^{-4}$, and $m^{2}=-3.5$.

where

$a_{l}=\frac{2^{l-1+\Delta_{-}} \Gamma\left(l+\frac{d}{2}+\frac{1}{2}\right) \Gamma\left(\Delta_{-}-\frac{d}{2}\right)}{\sqrt{\pi} \Gamma\left(l+\Delta_{-}\right)}, \quad b_{l}=\frac{2^{l-1+\Delta_{+}} \Gamma\left(l+\frac{d}{2}+\frac{1}{2}\right) \Gamma\left(\Delta_{+}-\frac{d}{2}\right)}{\sqrt{\pi} \Gamma\left(l+\Delta_{+}\right)}$.

It is then straightforward to check that the ratio of determinants

$$
\frac{\operatorname{det}_{\gamma_{1}}\left(-\square+m^{2}\right)}{\operatorname{det}_{\gamma_{2}}\left(-\square+m^{2}\right)}=\frac{\gamma_{1} p_{l}(\epsilon)+\epsilon p_{l}^{\prime}(\epsilon)}{\gamma_{2} p_{l}(\epsilon)+\epsilon p_{l}^{\prime}(\epsilon)}
$$

agrees exactly with the expected CFT answer on $\mathbf{S}^{d}$

$$
\frac{1+f_{1} g_{l}}{1+f_{2} g_{l}}
$$

where $g_{l}$ is the eigenvalue of the boundary Laplacian, (2.16). To be precise, we find the CFT answer on a $d$-sphere with $R=1 / \epsilon$, which is as expected since in writing the hyperbolic coordinates (4.28) we have normalized the boundary metric as $d \Omega_{d}^{2} / \epsilon^{2}$.

We have also performed numerical checks of our method for computing determinant ratios, in both Poincaré and hyperbolic coordinates . In hyperbolic coordinates, the boundary condition $\phi_{l}(\rho=2)=0$ selects (4.31) rather than the other solution to the hypergeometric equation, which blows up at $\rho=2$. The boundary condition $\gamma \phi_{l}(\epsilon)+\epsilon \phi_{l}^{\prime}(\epsilon)=0$ then quantizes $\nu$, giving a discrete set of eigenvalues that are multiplied together to compute the determinant. The determinants are infinite but their ratios are finite, converging after a few thousand eigenvalues.

As an example, we compare the numerical and analytical results for two different choices of $\gamma_{1}, \gamma_{2}$ in Table 1]. Figure 2] shows a Mathematica plot of the convergence of

$$
R \equiv \frac{\operatorname{det}_{\gamma_{1}}\left(-\square+m^{2}\right)}{\operatorname{det}_{\gamma_{2}}\left(-\square+m^{2}\right)}
$$


for Point B. The horizontal axis is the number of eigenvalues $n$ included in the computation, and the flat line is the analytical answer obtained from the boundary value trick. The numerical solution approaches the analytical solution like $1 / n$. For each set of parameters, the numerical solution in Table 1 was obtained by fitting the curve to a power law and extrapolating to $n \rightarrow \infty$.

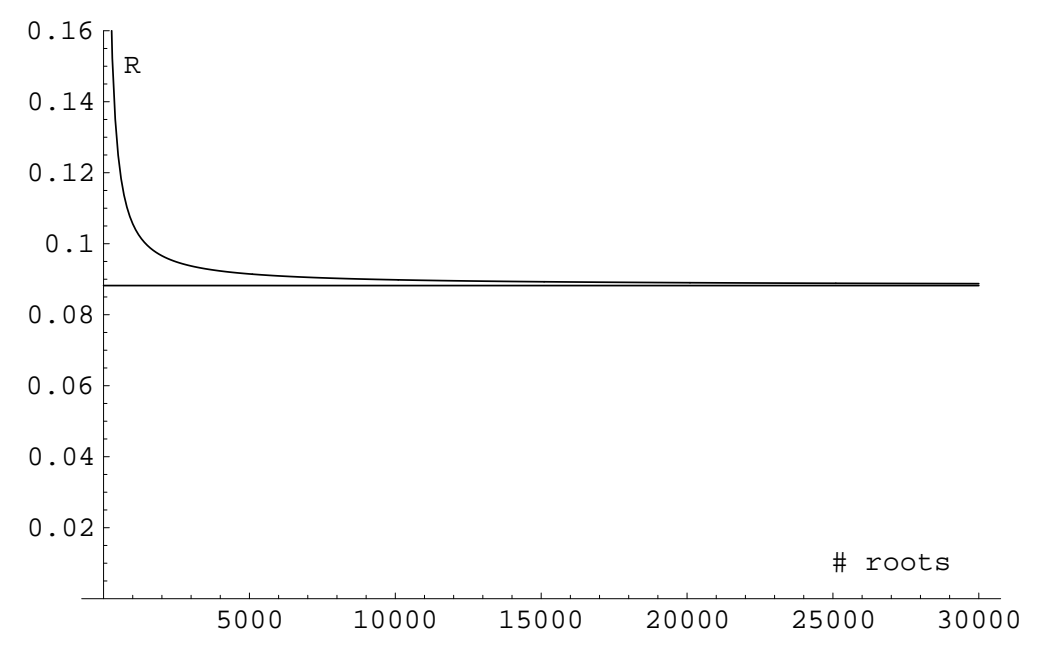

Figure 2: Numerical value of $R$ in eq. 4.37) versus the number of roots included in the computation. The curve converges nicely to the horizontal line (the analytical answer). 


\section{5. $\Delta_{-}$boundary conditions and 1PI diagrams}

The auxiliary field trick was used in $[3$ to show that in the large $N$ boundary theory, the generating functional $W_{I R}[\tilde{J}]$ at the IR fixed point is the Legendre transform of the generating functional $W_{U V}[J]$ at the UV fixed point (see [19] for a recent discussion). This agrees with the recipe postulated by Klebanov and Witten 23] for the evaluation of AdS/CFT correlators with $\Delta_{-}$boundary conditions: namely one is instructed to first evaluate the correlators with $\Delta_{+}$boundary conditions, using the standard algorithm, and then to perform a Legendre transform with respect to the source $\tilde{J}$ for the operator $\mathcal{O}_{\Delta_{+}}$. While this is a consistent state of affairs, it would be more satisfactory to treat the two boundary conditions on a more symmetric footing, and have an intrinsic algorithm that directly computes AdS/CFT correlators with $\Delta_{-}$boundary conditions.

Actually, we have already stated such an algorithm in section 3: evaluate the on-shell bulk action as a functional of the boundary source $\phi_{b} \equiv J$ of eq. (3.9), imposing the mixed boundary condition with $\gamma=-\Delta_{-}$. In section 3 we were dealing with two-point functions, and thus restricting to the quadratic part of the action. For higher point function, we proceed in the standard way [27, 28, treating the interactions perturbatively, and computing AdS/CFT correlators as sums of diagrams built with bulk-to-boundary propagators, bulk-to-bulk propagators and bulk vertices (see e.g. [31]). Our algorithm is simply stated: use the propagators appropriate to $\Delta_{-}$boundary conditions, and otherwise proceed as usual.

Let us take a closer look at the propagators, contrasting regular and irregular boundary conditions. It will be convenient to work in momentum space. The bulk-to-boundary propagators for $\Delta_{+}$and $\Delta_{-}$are given by (3.15), respectively with $\gamma=\infty$ and $\gamma=-\Delta_{-}$,

$$
\begin{aligned}
& P_{\Delta_{+}}(k)=\frac{\psi\left(k, x_{0}\right)}{\psi(k, \epsilon)} \stackrel{\epsilon \rightarrow 0}{\longrightarrow} \frac{2^{1-\nu}}{\Gamma(\nu)}(\epsilon k)^{\nu} x_{0}^{\nu} \mathcal{K}_{\nu}\left(k x_{0}\right), \\
& P_{\Delta_{-}}(k)=\frac{\psi\left(k, x_{0}\right)}{-\Delta_{-} \psi(\epsilon, k)+\epsilon \partial \psi(k, \epsilon)} \stackrel{\epsilon \rightarrow 0}{\longrightarrow} \frac{2^{1+\nu}}{2 \nu \Gamma(-\nu)}(\epsilon k)^{-\nu} x_{0}^{\nu} \mathcal{K}_{\nu}\left(k x_{0}\right),
\end{aligned}
$$

where we have reabsorbed the prefactor of $1 / \gamma \rightarrow 0$ in $P_{\Delta_{+}}$in a redefinition of the source. The two propagators have the same functional form in $x_{0}$. This is bound to happen since there is a unique solution of the wave equation regular in 
the interior. They differ however by an important $k$ dependent factor,

$$
\frac{P_{\Delta_{+}}(k)}{P_{\Delta_{-}}(k)} \sim k^{2 \nu} .
$$

The bulk-to-bulk propagators are

$$
\begin{aligned}
& G_{\Delta_{+}}\left(k ; x_{0}, y_{0}\right)=\left(x_{0} y_{0}\right)^{d / 2} I_{\nu}\left(k z_{0}^{<}\right) \mathcal{K}_{\nu}\left(k z_{0}^{>}\right) \\
& G_{\Delta_{-}}\left(k ; x_{0}, y_{0}\right)=\left(x_{0} y_{0}\right)^{d / 2} I_{-\nu}\left(k z_{0}^{<}\right) \mathcal{K}_{\nu}\left(k z_{0}^{>}\right),
\end{aligned}
$$

where $z_{0}^{<}$is the smaller of the radial coordinates $x_{0}, y_{0}$ and $z_{0}^{>}$is the larger. ${ }^{8}$ We observe that their difference takes the factorized form

$$
G_{\Delta_{+}}\left(k, x_{0}, y_{0}\right)-G_{\Delta_{-}}\left(k, x_{0}, y_{0}\right)=-\frac{2 \sin (\pi \nu)}{\pi}\left(x_{0} y_{0}\right)^{d / 2} \mathcal{K}_{\nu}\left(k x_{0}\right) \mathcal{K}_{\nu}\left(k y_{0}\right)
$$

It is not immediately obvious that exchanging $\Delta_{+}$with $\Delta_{-}$propagators is equivalent to the Legendre transform recipe stated above. We are now going to show that this is the case. We will briefly illustrate our claim for 4pt functions - it is easy to fill in the details and generalize the argument to arbitrary $n$-point functions. As is familiar, the Legendre transform of the generator of connected correlation functions is the generator of one particle irreducible (1PI) correlation functions, with external legs amputated. We use the following diagrammatic notation for the action of the Legendre transform on a 4 pt function, ${ }^{9}$

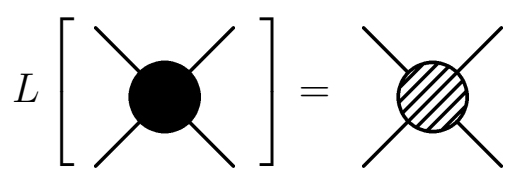

Black dots represent connected correlators and hatched dots represent 1PI correlators. The connected 4-point function can be expanded with 1PI vertices and

\footnotetext{
${ }^{8}$ For simplicity, we have written the expressions of the bulk-to-bulk propagators in the limit $\epsilon \rightarrow 0$. This is legitimate in most cases. For special correlators (for example extremal correlators 32]), if one is interested in their exact normalization, it may be necessary to use the more cumbersome expressions with finite $\epsilon$ and take the limit $\epsilon \rightarrow 0$ at the end of the calculation.

${ }^{9}$ Of course, we are taking the Legendre transform of a generating functional, not of a single correlator. What we mean by the Legendre transform of a 4 pt function is actually the fourth functional derivative of the Legendre-transformed generating functional.
} 
full propagators,

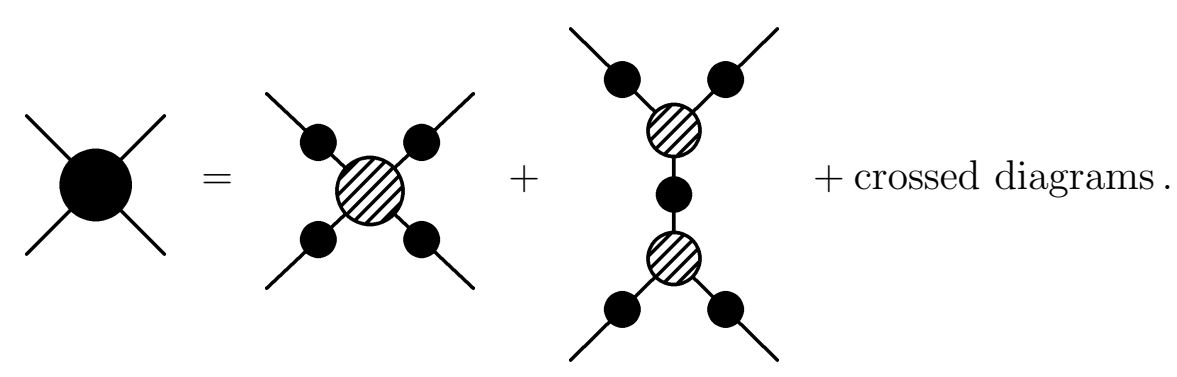

Therefore we can take the Legendre transform of a correlator by subtracting reducible parts and removing the external legs,

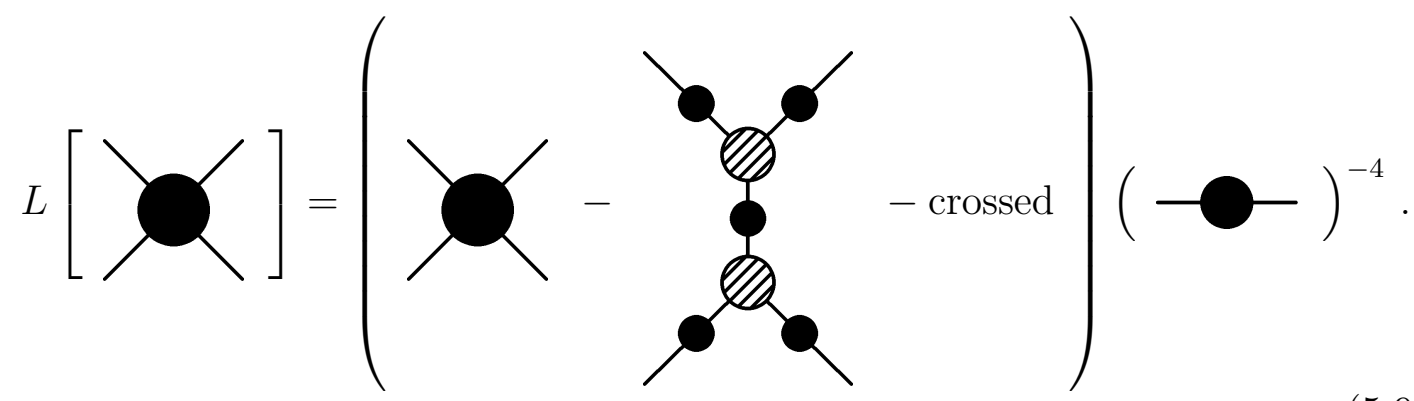

On the other hand, AdS diagrams are drawn in the usual way, with a circle on the outside that represents the boundary. The statement of the AdS/CFT correspondence is

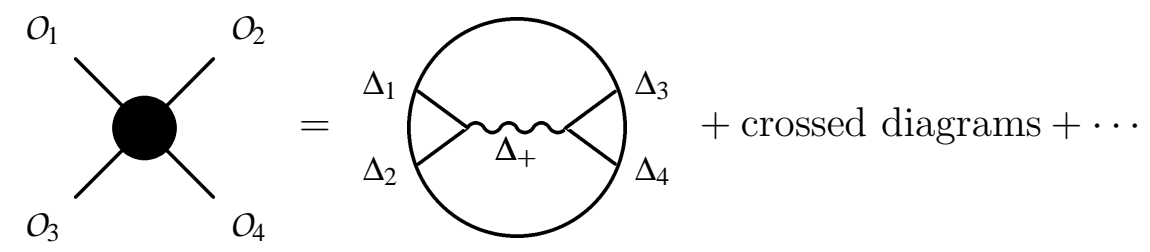

The dots stand for diagrams without explicit dependence on the field dual to $\mathcal{O}_{\Delta_{+}}$. Here it is understood that the AdS diagrams on the rhs are built with propagators obeying the "regular" (Dirichlet) boundary conditions. We wish to show that taking the Legendre transform is equivalent to using instead the "irregular" propagators. The external operators $\mathcal{O}_{\Delta_{i}}, i=, 1 \ldots, 4$ need not be the same as $\mathcal{O}_{\Delta_{+}}$. Let us first assume for simplicity that they are all different from $\mathcal{O}_{\Delta_{+}}$. We are then instructed to take the Legendre transform with respect to the source of $\mathcal{O}_{\Delta_{+}}$, leaving the operators $\mathcal{O}_{\Delta_{i}}$ unchanged. The identity (5.9), where external legs are removed on the right-hand side, assumed that only a single operator was involved so all of the external legs belonged to that operator. In the present case, the proper prescription is to leave the external legs intact. 
Diagrammatically, the statement is

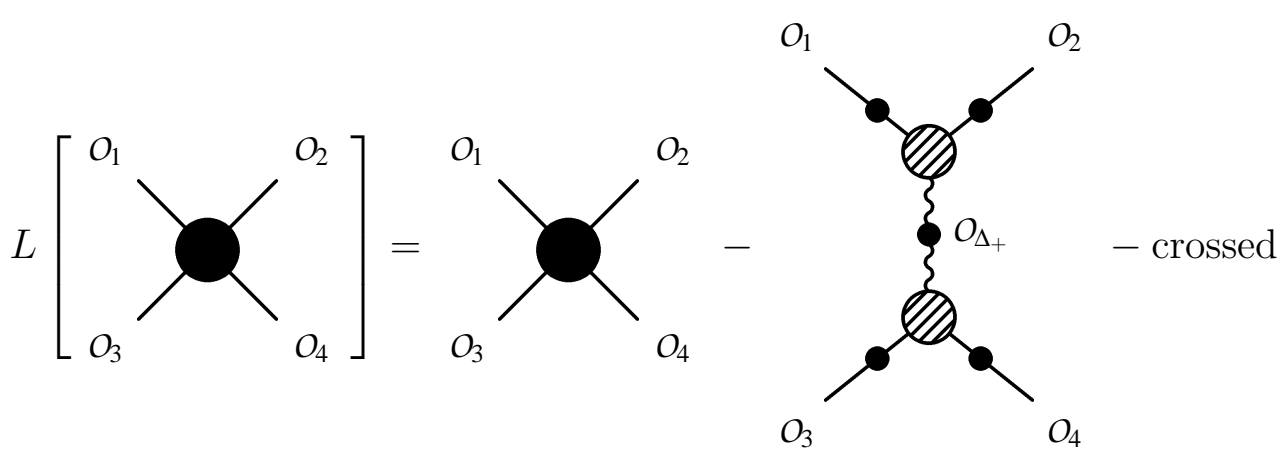

In the second diagram on the rhs, the internal wavy line with a small black dot represents the $\mathcal{O}_{\Delta_{+}}$2pt correlator, $\left\langle\mathcal{O}_{\Delta_{+}}(k) \mathcal{O}_{\Delta_{+}}(k)\right\rangle \sim k^{2 \nu}$, while the external solid lines with little black dots are the 2 pt correlators of the $\mathcal{O}_{\Delta_{i}}$ operators. In momentum space the second diagram can be also be written

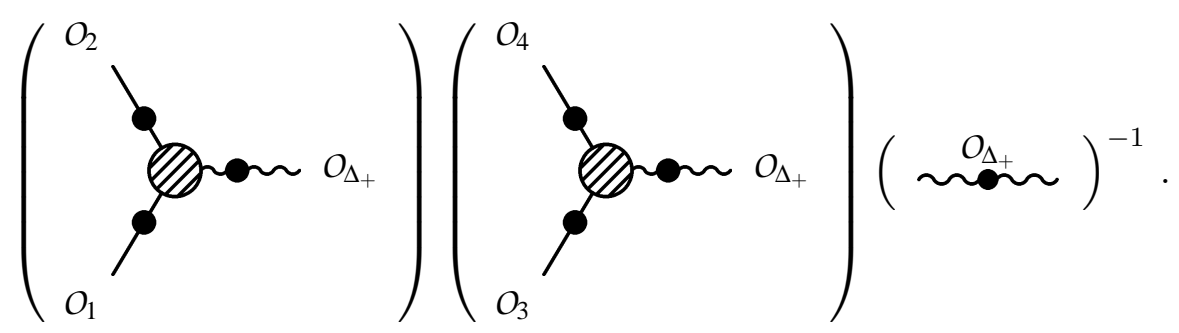

Translated into AdS language, this says (we are being a little schematic and dropping constant factors)

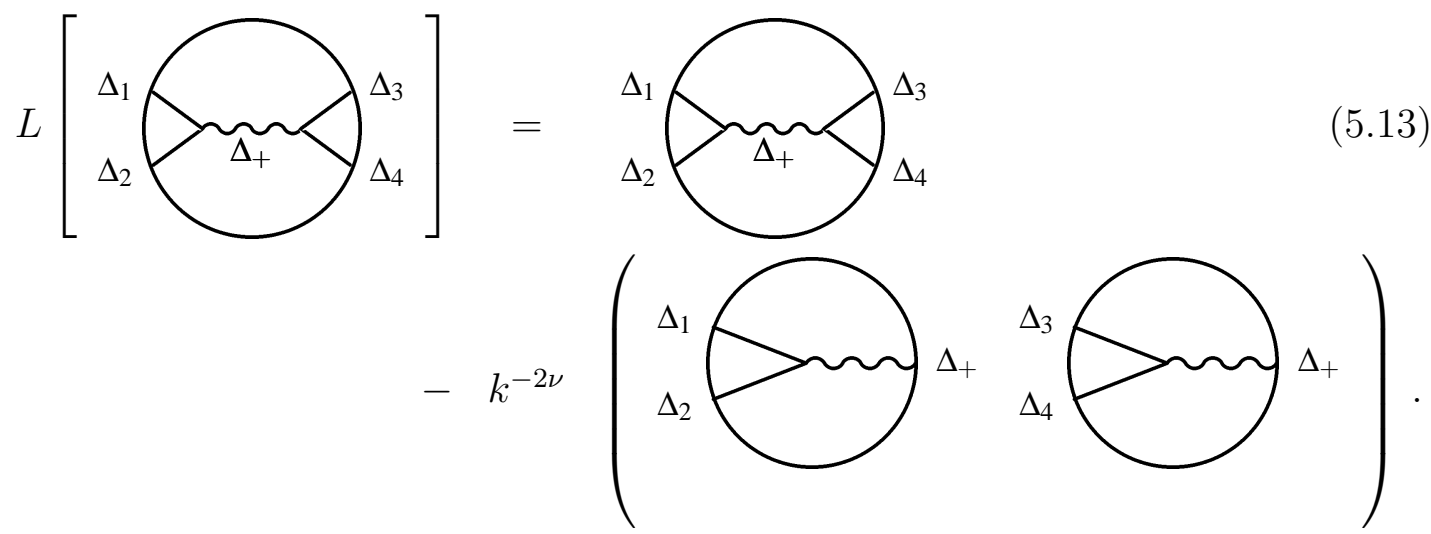

We need to show that the right-hand side of (5.13) is actually equal to the exchange diagram with a bulk $\Delta_{-}$propagator, so that

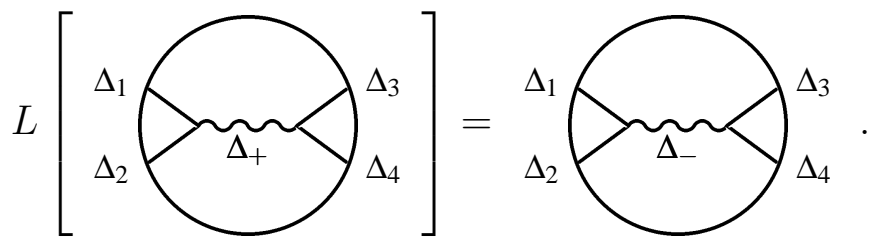


Indeed, let us evaluate the difference of exchange diagrams with regular and irregular boundary conditions,

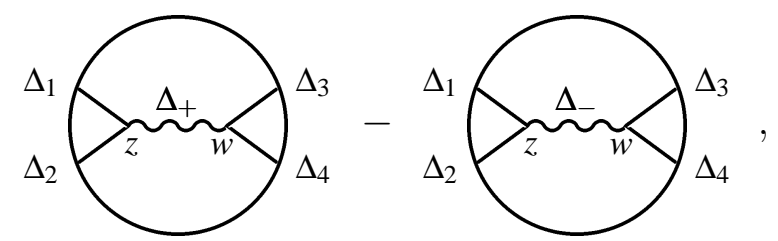

whose analytic expression is

$$
\begin{aligned}
& \int \frac{d z_{0}}{z_{0}^{d+1}} \int \frac{d w_{0}}{w_{0}^{d+1}} P_{\Delta_{1}}\left(\vec{p}_{1}, z_{0}\right) P_{\Delta_{2}}\left(\vec{p}_{2}, z_{0}\right) P_{\Delta_{3}}\left(\vec{p}_{3}, w_{0}\right) P_{\Delta_{4}}\left(\vec{p}_{4}, w_{0}\right) \\
& \times\left[G_{\Delta_{+}}\left(\vec{k}, z_{0}, w_{0}\right)-G_{\Delta_{-}}\left(\vec{k}, z_{0}, w_{0}\right)\right] .
\end{aligned}
$$

Using (15.6), the integrand is proportional to

$$
P_{\Delta_{1}}\left(\vec{p}_{1}, z_{0}\right) P_{\Delta_{2}}\left(\vec{p}_{2}, z_{0}\right) P_{\Delta_{3}}\left(\vec{p}_{3}, w_{0}\right) P_{\Delta_{4}}\left(\vec{p}_{4}, w_{0}\right)\left[z_{0}^{d / 2} \mathcal{K}_{\nu}\left(k z_{0}\right) w_{0}^{d / 2} \mathcal{K}_{\nu}\left(k w_{0}\right)\right]
$$

The difference between the bulk propagators (the term in brackets) is seen to be identical to product of two bulk-to-boundary propagators $P_{\Delta_{+}}$, missing the factors of $k^{\nu}$, so we have found

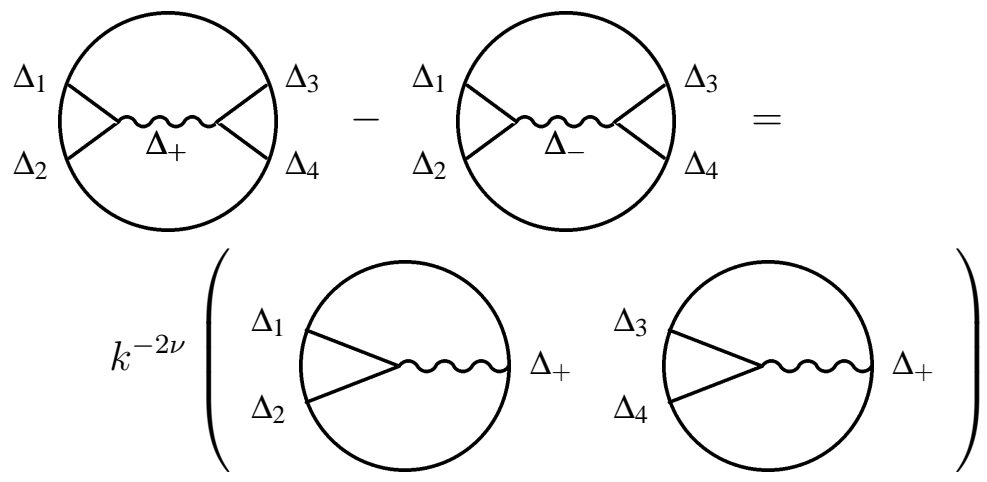

Comparing to (5.13), this completes the proof of (5.14).

Finally, let us consider the case where some of the external operators $\mathcal{O}_{\Delta_{i}}$ are also equal to $\mathcal{O}_{\Delta_{+}}$. In taking the Legendre transform, we are now further instructed to amputate each external leg involving a $\mathcal{O}_{\Delta_{+}}$, see (5.9). In momentum space, this amounts to removing factors of $\left\langle\mathcal{O}_{\Delta_{+}}\left(k_{i}\right) \mathcal{O}_{\Delta_{+}}\left(-k_{i}\right)\right\rangle \sim k_{i}^{-2 \nu}$ for each external momentum $k_{i}$ corresponding to a boundary insertion of $\mathcal{O}_{\Delta_{+}}$. But this is exactly equivalent to changing boundary conditions for the bulk-to-boundary propagators, $P_{\Delta_{+}}\left(k_{i}\right) \rightarrow P_{\Delta_{-}}\left(k_{i}\right)$ ! This concludes the argument that correlation 
functions computed with $\Delta_{-}$propagators are precisely the Legendre transform of correlations functions computed with $\Delta_{+}$propagators.

\section{Acknowledgments}

It is a pleasure to thank Igor Klebanov for useful discussions at various stages of this project, and Dan Freedman and Igor Klebanov for critical reading of the manuscript. This material is based in part upon work supported by the National Science Foundation Grant No. PHY-0243680. Any opinions, findings, and conclusions or recommendations expressed in this material are those of the authors and do not necessarily reflect the views of the National Science Foundation.

\section{A. One-loop effective potential in AdS}

The change in the central charge of the bulk theory was computed by Gubser and Mitra 2]. We will review the computation here, using a different method to regulate the infinities but otherwise following their calculation.

We are interested in the one-loop AdS effective action, ${ }^{10}$

$$
W=-\frac{i}{2} \operatorname{Tr} \log \left(-\square+m^{2}\right)
$$

Taking the derivative of this expression,

$$
\frac{\partial W}{\partial m^{2}}=\frac{i}{2} \operatorname{Tr}\left(\frac{1}{-\square+m^{2}}\right) .
$$

Therefore the logarithm in the effective action can be written as an integral,

$$
W=-\frac{i}{2} \operatorname{Tr} \int d m^{2} G
$$

where $G$ is the scalar propagator, defined as the inverse of the wave operator. The trace is an integral $\int d^{d+1} x \sqrt{g}$ over the AdS bulk. The maximal symmetry of AdS implies that $G(x, x)$ is independent of $x$, so that the trace contributes only the overall AdS volume $\mathrm{Vol}_{A d S}$. We can then define the effective potential $V\left(m^{2}\right)$ as

$$
V\left(m^{2}\right) \equiv \frac{W\left(m^{2}\right)}{\operatorname{Vol}(A d S)}=-\frac{i}{2} \int_{m^{2}}^{\infty} d \tilde{m}^{2} G\left(x, x ; \tilde{m}^{2}\right) .
$$

\footnotetext{
${ }^{10}$ In this appendix we adopt Lorentzian signature.
} 
A more rigorous derivation using the DeWitt-Schwinger representation of the propagator is given in [33], Section 6.1.

Generally, both the integrand and the integral over $\tilde{m}^{2}$ are divergent. Since we are only interested in the change in the effective potential, we can regulate the UV divergence in the integrand by computing the difference in $V$ going from regular $(+)$ to irregular $(-)$ boundary conditions,

$$
V_{+}-V_{-}=-\frac{i}{2} \int_{m^{2}}^{\infty} d \tilde{m}^{2}\left(G_{\tilde{\Delta}_{+}}(x, x)-G_{\tilde{\Delta}_{-}}(x, x)\right) .
$$

The integral, however, still diverges. To regulate it, the authors of [2] split the integral into two parts,

$V_{+}\left(m^{2}\right)-V_{-}\left(m^{2}\right)=\frac{i}{2} \int_{m_{B F}^{2}}^{m^{2}} d \tilde{m}^{2}\left(G_{\tilde{\Delta}_{+}}(x, x)-G_{\tilde{\Delta}_{-}}(x, x)\right)+\left[V_{+}\left(m_{B F}^{2}\right)-V_{-}\left(m_{B F}^{2}\right)\right]$.

The integral is finite. The second term is the change in the effective potential for a field with mass saturating the Breitenlohner-Freedman bound $m_{B F}^{2} L^{2}=-d^{2} / 4$. This is the lowest mass for which a scalar field can be quantized consistently on AdS, and corresponds to a boundary operator with scaling dimension $\Delta=d / 2$.

It seems reasonable to expect that the change in the effective potential to vanish at the $\mathrm{BF}$ bound, leaving only the finite terms in equation (A.6). A heuristic argument is as follows 2]. The expression for the mode-sum vacuum energy in the Hamiltonian formalism is

$$
E_{v a c}=\frac{1}{2} \sum_{k} \omega_{k}
$$

For a scalar field, the mode frequencies in global AdS coordinates are $\omega=l+$ $2 n+\Delta_{ \pm}$(see e.g. [34]). Therefore, at the BF bound where $\Delta_{+}=\Delta_{-}$, regular and irregular modes contribute equally to the vacuum energy. Thus we have $E_{v a c}^{+}\left(m_{B F}^{2}\right)-E_{v a c}^{-}\left(m_{B F}^{2}\right)=0$. However, there is a potential loophole in this argument: the quantity $E_{v a c}^{+}-E_{v a c}^{-}$cannot necessarily be identified with the change in the effective potential $V_{+}-V_{-}$. These two quantities are equal in flat space, but they can be different in curved space if the $g_{t t}$ component of the metric is non-trivial (see e.g. 35]). They are in fact different in our case, as can be verified by performing the sum explicitly with an exponential or zeta-function regulator.

Therefore, we will use a different method that allows us to compute $V$ directly from (A.5). We work in global coordinates,

$$
d s^{2}=L^{2}\left[-\sec ^{2} \rho d t^{2}+\sec ^{2} \rho d \rho^{2}+\tan ^{2} \rho d \Omega_{d-1}^{2}\right]
$$


We begin by writing the scalar propagator as a mode-sum,

$$
i G_{\Delta_{ \pm}}\left(x, x^{\prime}\right)=\theta\left(x, x^{\prime}\right) \sum_{n, l, m} \Psi_{n l m}^{\Delta_{ \pm}^{*}}(x) \Psi_{n l m}^{\Delta_{ \pm}}\left(x^{\prime}\right)+\theta\left(x^{\prime}, x\right) \sum_{n, l, m} \Psi_{n l m}^{\Delta_{ \pm}^{*}}\left(x^{\prime}\right) \Psi_{n l m}^{\Delta_{ \pm}}(x)
$$

where the sum is over all normalized modes of the scalar field and $\theta\left(x, x^{\prime}\right)$ is the AdS time-ordering operator. Note that the modes are taken on-shell, $\omega=$ $l+2 n+\Delta_{ \pm}$. This is easily seen to be a valid propagator by applying the wave operator $-\square+m^{2}$. Acting on the $\theta$-functions gives a delta function $\delta\left(x-x^{\prime}\right)$, and the equation of motion for $\Psi_{n l m}^{\Delta_{ \pm}}$gives zero for $x \neq x^{\prime}$.

Plugging in the scalar modes (from e.g. 34]), we have

$$
\begin{aligned}
i G_{\Delta}\left(x, x^{\prime}\right)= & \sum_{n, l, m} N_{\Delta}^{2} e^{-i \omega\left(t-t^{\prime}\right)} Y_{l m}(\Omega) Y_{l m}\left(\Omega^{\prime}\right)(\sin \rho)^{l}(\cos \rho)^{\Delta}\left(\sin \rho^{\prime}\right)^{l}\left(\cos \rho^{\prime}\right)^{\Delta} \\
& \times P_{n}^{(l+d / 2-1, \Delta-d / 2)}(\cos 2 \rho) P_{n}^{(l+d / 2-1, \Delta-d / 2)}\left(\cos 2 \rho^{\prime}\right),
\end{aligned}
$$

where $N_{\Delta}^{2}$ is a normalization constant that can be computed from the scalar inner product and $P_{n}^{a, b}$ is a Jacobi polynomial. The maximal symmetry of AdS allows us translate to $x^{\prime}=0$, considerably simplifying the sum by ensuring that only $l=0, m=0$ modes will contribute. All other terms are zero by virtue of the $\left(\sin \rho^{\prime}\right)^{l}$ factor. $Y_{00}(\Omega)$ is constant and normalized on the sphere $S^{d-1}$, so the propagator is

$$
i G_{\Delta}(x)=\frac{(\cos \rho)^{\Delta}}{\operatorname{Vol}\left(S^{d-1}\right)} \sum_{n=0}^{\infty} N_{\Delta}^{2} P_{n}^{(d / 2-1, \Delta-d / 2)}(1) P_{n}^{(d / 2-1, \Delta-d / 2)}(\cos 2 \rho) e^{-i(2 n+\Delta) t} .
$$

This can be summed to give the usual expression for the propagator in global coordinates. To regulate the coincidence limit of (A.9), which does not depend on $x$, we set $\rho=\rho^{\prime}$ and $\Omega=\Omega^{\prime}$ but maintain $t \neq t^{\prime}$ until the very end. After interchanging the order of the limit, sum, and integral, this gives

$$
V\left(m^{2}\right)=-\lim _{t \rightarrow 0} \frac{1}{4 L^{d} \pi^{d / 2} \Gamma\left(\frac{d}{2}\right)} \sum_{n=0}^{\infty} \int_{\nu^{2}}^{\infty} d \tilde{\nu}^{2} \frac{\Gamma\left(n+\frac{d}{2}\right) \Gamma\left(n+\frac{d}{2}+\tilde{\nu}\right)}{\Gamma(n+1) \Gamma(n+1+\tilde{\nu})} e^{-i\left(2 n+\frac{d}{2}+\tilde{\nu}\right) t} .
$$

Everything but the final limit is finite. To regulate the $t \rightarrow 0$ divergence, we expand the answer as a power series in $t$ and take the difference $V_{+}-V_{-}$before taking the limit. The result is finite.

For even $d$, the integral is simple and we obtain the following:

$$
\text { - } \mathbf{d}=\mathbf{2}: \quad V_{+}-V_{-}=-\frac{\nu^{3}}{6 \pi L^{2}}
$$


- $\mathbf{d}=4: \quad V_{+}-V_{-}=\frac{1}{12 \pi^{2} L^{4}}\left(-\frac{\nu^{3}}{3}+\frac{\nu^{5}}{5}\right)$

- $\mathbf{d}=\mathbf{6}: \quad V_{+}-V_{-}=\frac{1}{120 \pi^{3} L^{6}}\left(-\frac{4}{3} \nu^{3}+\nu^{5}-\frac{\nu^{7}}{7}\right)$

- $\mathbf{d}=\mathbf{8}: \quad V_{+}-V_{-}=\frac{1}{1680 \pi^{4} L^{8}}\left(-12 \nu^{3}+\frac{49}{5} \nu^{5}-2 \nu^{7}+\frac{\nu^{9}}{9}\right)$

These results agree with [2, thereby confirming their a priori assumption that $V_{+}\left(m_{B F}^{2}\right)=V_{-}\left(m_{B F}^{2}\right)$. They are also compatible with the calculation in 36] of the (Euclidean) one-loop effective potential by zeta-function regularization, if one assumes that we can obtain $V_{-}$by simply replacing $\Delta_{+} \rightarrow \Delta_{-}$in the final expressions for $V_{+}$quoted in [36].

\section{References}

[1] E. Witten, "Multi-trace operators, boundary conditions, and AdS/CFT correspondence," hep-th/0112258.

[2] S. S. Gubser and I. Mitra, "Double-trace operators and one-loop vacuum energy in AdS/CFT," Phys. Rev. D67 (2003) 064018, hep-th/0210093.

[3] S. S. Gubser and I. R. Klebanov, "A universal result on central charges in the presence of double-trace deformations," Nucl. Phys. B656 (2003) 23, hep-th/0212138

[4] O. Aharony, M. Berkooz and E. Silverstein, "Multiple-trace operators and non-local string theories," JHEP 0108, 006 (2001) arXiv:hep-th/0105309. "Non-local string theories on $\operatorname{AdS}(3)$ x $\mathrm{S}(3)$ and stable non-supersymmetric backgrounds," Phys. Rev. D 65, 106007 (2002) arXiv:hep-th/0112178.

[5] M. Berkooz, A. Sever and A. Shomer, "Double-trace deformations, boundary conditions and spacetime singularities," JHEP 0205, 034 (2002) arXiv:hep-th/0112264.

[6] W. Muck, "An improved correspondence formula for AdS/CFT with multi-trace operators," Phys. Lett. B 531, 301 (2002) arXiv:hep-th/0201100.

[7] P. Minces, "Multi-trace operators and the generalized AdS/CFT prescription," Phys. Rev. D 68, 024027 (2003) arXiv:hep-th/0201172.

[8] A. C. Petkou, "Boundary multi-trace deformations and OPEs in AdS/CFT correspondence," JHEP 0206, 009 (2002) arXiv:hep-th/0201258. 
[9] A. Sever and A. Shomer, "A note on multi-trace deformations and AdS/CFT," JHEP 0207, 027 (2002) arXiv:hep-th/0203168.

[10] J. L. F. Barbon, "Multitrace AdS/CFT and master field dynamics," Phys. Lett. B 543, 283 (2002) arXiv:hep-th/0206207.

[11] I. R. Klebanov and A. M. Polyakov, "AdS dual of the critical O(N) vector model," Phys. Lett. B 550, 213 (2002) arXiv:hep-th/0210114.

[12] L. Girardello, M. Porrati and A. Zaffaroni, "3-D interacting CFTs and generalized Higgs phenomenon in higher spin theories on AdS," Phys. Lett. B 561, 289 (2003) arXiv:hep-th/0212181.

[13] S. Nojiri and S. D. Odintsov, "The one-loop vacuum energy and RG flow induced by double-trace operators in AdS/CFT and dS/CFT correspondence," Phys. Rev. D 69, 023511 (2004) arXiv:hep-th/0302054.

[14] J. Troost, "A note on causality in the bulk and stability on the boundary," Phys. Lett. B 578, 210 (2004) arXiv:hep-th/0308044.

[15] M. J. Strassler, "Non-supersymmetric theories with light scalar fields and large hierarchies," arXiv:hep-th/0309122.

[16] J. L. F. Barbon and C. Hoyos, "AdS/CFT, multitrace deformations and new instabilities of nonlocal string theories," JHEP 0401, 049 (2004) arXiv:hep-th/0311274.

[17] P. Minces, "Bound states in the AdS/CFT correspondence," Phys. Rev. D 70, 025011 (2004) arXiv:hep-th/0402161.

[18] O. Aharony, M. Berkooz and B. Katz, "Non-local effects of multi-trace deformations in the AdS/CFT correspondence," JHEP 0510, 097 (2005) arXiv:hep-th/0504177.

[19] S. Elitzur, A. Giveon, M. Porrati and E. Rabinovici, "Multitrace deformations of vector and adjoint theories and their holographic duals," arXiv:hep-th/0511061

[20] T. Hertog and G. T. Horowitz, "Designer gravity and field theory effective potentials," Phys. Rev. Lett. 94, 221301 (2005) arXiv:hep-th/0412169.

[21] E. Barnes, E. Gorbatov, K. Intriligator, M. Sudano and J. Wright, "The exact superconformal R-symmetry minimizes tau(RR)," Nucl. Phys. B 730, 210 (2005) arXiv:hep-th/0507137. 
[22] D. Martelli, J. Sparks and S. T. Yau, "The geometric dual of a-maximisation for toric Sasaki-Einstein manifolds," arXiv:hep-th/0503183.

[23] I. R. Klebanov and E. Witten, "AdS/CFT correspondence and symmetry breaking," Nucl. Phys. B556 (1999) 89, hep-th/9905104.

[24] P. Breitenlohner and D. Z. Freedman, "Positive energy in anti-de Sitter backgrounds and gauged extended supergravity," Phys. Lett. B115 (1982) 197.

[25] D. Z. Freedman, S. D. Mathur, A. Matusis, and L. Rastelli, "Correlation functions in the CFT $(d) / \operatorname{AdS}(d+1)$ correspondence," Nucl. Phys. B546 (1999) 96, hep-th/9804058.

[26] M. Henningson and K. Skenderis, "The holographic Weyl anomaly," JHEP 07 (1998) 023, hep-th/9806087.

[27] S. S. Gubser, I. R. Klebanov, and A. M. Polyakov, "Gauge theory correlators from non-critical string theory," Phys. Lett. B428 (1998) 105, hep-th/9802109

[28] E. Witten, "Anti-de Sitter space and holography," Adv. Theor. Math. Phys. 2 (1998) 253, hep-th/9802150.

[29] S. Coleman, Aspects of Symmetry: Selected Erice Lectures. Cambridge University Press, Cambridge, 1985.

[30] K. Kirsten and A. J. McKane, "Functional determinants for general Sturm-Liouville problems," math-ph/0403050.

[31] E. D’Hoker, D. Z. Freedman, S. D. Mathur, A. Matusis and L. Rastelli, "Graviton exchange and complete 4-point functions in the AdS/CFT correspondence," Nucl. Phys. B 562, 353 (1999) arXiv:hep-th/9903196.

[32] E. D’Hoker, D. Z. Freedman, S. D. Mathur, A. Matusis and L. Rastelli, "Extremal correlators in the AdS/CFT correspondence," arXiv:hep-th/9908160.

[33] Birrell and Davies, Quantum Fields in Curved Space. Cambridge University Press, Cambridge, 1982.

[34] V. Balasubramanian, P. Kraus, and A. E. Lawrence, "Bulk vs. boundary dynamics in anti-de Sitter spacetime," Phys. Rev. D59 (1999) 046003, hep-th/9805171. 
[35] R. Camporesi and A. Higuchi, "Stress energy tensors in anti-de Sitter space-time," Phys. Rev. D45 (1992) 3591.

[36] M. M. Caldarelli, "Quantum scalar fields on anti-de Sitter spacetime," Nucl. Phys. B549 (1999) 499, hep-th/9809144. 